\title{
Translation of the Proper Nouns in Legal English
}

\author{
Gang Tang \\ Wuhan University Institute of International Law, Wuhan, China
}

\begin{abstract}
Proper nouns have posed a challenge to translators of legal English for a long time. Legal English has the characteristics of formality, accuracy, ambiguity, professional, etc. These characteristics have made it more difficult for translation of the proper nouns in legal English. However, due to not paying enough attention to translation of the proper nouns in legal English, translators engaged in legal translation often make a lot of mistakes during the process of translation. Generally speaking, names of people or places are transliterated, while names of institutions or books are semantic translated. Transliteration should follow the principle of conformity to the original and the principle of convention, the former is mainly applied to proper nouns of non-English origin, while the latter concerning established wording or phrasing. In order to translate the proper nouns in legal English better, firstly, we should be aware of the difficulties of translation of the proper nouns in legal English; secondly, we should try our best to accumulate extensive legal knowledge and improve our translation skills; thirdly, we should make full use of reference books and master the skills of Internet application; last but not the least, we should ask for advice from others.
\end{abstract}

Index Terms-proper nouns, legal English, semantic translation, transliteration

\section{INTRODUCTION}

Translation of proper nouns once was a hot topic in Chinese translation field. Many translators have expressed their opinions on it since modern times. Even in the current, there are still a lot of articles discussing translation of proper nouns. Some translation courses are also equipped with the special chapters to analyze translation of names of people or places and other proper nouns. These articles and courses have analyzed proper nouns' constitution characteristics, established the basic principles of translation of proper nouns, and proposed concrete translation methods, which have practical guidance to translators(Zhang, 2008).However, these scholars have failed to notice the new changes of foreign language in proper nouns, so they have been out of touch with the current translation practice. More importantly, the research results related to translation of the proper nouns in legal English are not very common. It seems that nobody shows any interests in the research area of discussion of translation of proper nouns combining the features of legal English (Li, 2007).The academic field lacks of systematic and comprehensive discussion and research on classifications, characteristics, difficulties of translation of proper nouns in legal English, translation methods, requirements for translators' translation of proper nouns in legal English and other problems. Therefore, it is still necessary to further discuss the problem of how to deal with proper nouns in legal translation.

This article takes "translation of the proper nouns in legal English" as the entry point and attempts to research and discuss the types of proper nouns in legal English, difficulties for translation of the proper nouns in legal English, methods of translating the proper nouns in legal English and requirements for translators' translation of proper nouns in legal English and other questions, which undoubtedly has important theories value and practical significance.

Firstly, this article classifies the types of proper nouns in legal English; secondly, it analyzes the difficulties of translation of proper nouns in legal English; thirdly, it discusses the methods of translation of proper nouns in legal English, lastly, it proposes some suggestions and requirements for translators' translation of proper nouns in legal English.

The article analyzes translation of the proper nouns in legal English. It includes five parts, which mainly includes: introduction, types of proper nouns in legal English, characteristics of legal English, difficulties for translation of the proper nouns in legal English, methods of translation of the proper nouns in legal English, requirements for translators' translation of proper nouns in legal English.

\section{TyPeS OF Proper NOUNS IN LEGAL ENGLISH}

A proper noun is a noun representing a unique entity, as distinguished from a common noun, which describes a class of entities. In English, proper nouns are not normally preceded by an article or other limiting modifier, and are used to denote a particular person, place, or object without regard to any descriptive meaning the word or phrase may have $(\mathrm{Li}$, 2009).There are many different types of proper nouns in legal English originated from different language or times, which includes names of people, names of books, names of institutions, names of official position, etc(Peter, 2001).The different types of proper nouns represent almost all the constitute characteristics of proper nouns in legal English. As to their classification, it can be divided into the following types.

Firstly, names of people. In legal English, names of people include jurists and scholars, some of which are familiar to us. For instance, Plato, Cicero, Montesquieu, Paine, Hugo Grotius and so on. Secondly, names of books. Names of 
books are a type of proper nouns in legal English. For example, Chinese Journal of Law, Law Review, On the Laws, the Spirits of Laws, Civil Law, Criminal Law, International Law and so on. Thirdly, names of institutions. For example, Ministry of Public Security, Ministry of Supervision, Office of Legislative Affairs, Maritime Court, Basic People's Court and so on. Fourthly, names of official position. Names of official position are also a type of proper nouns in legal English, for example, judges, bailiff, court police, third party, procurator, presiding judge, chief justice, chief procurator and so on.

The above different types of proper nouns in legal English have their own characteristics and have posed a challenge to translation of legal English for long. Besides, with the development of language, new proper nouns will appear. Therefore, we need to pay more attention to translation of proper nouns in legal English.

\section{CHARACTERISTICS OF LEGAL ENGLiSH}

Before we discuss the translation of the proper nouns in legal English, we need to talk about characteristics of legal English. If we want to better translate the proper nouns in legal English, it is need to study characteristics of legal English. Only having a better understanding of characteristics of legal English, can we better translate the proper nouns in legal English. In conclusion, legal English has the characteristics of formality, accuracy, ambiguity, professional.

\section{A. Formality}

Legal English is used by the legal workers of English-speaking countries; it is a kind of English for Special Purpose. Their words have their own significant characteristics. The used words in legal English are formal and solemn. Oral English, slang and dialects can not be used under any circumstances. Making proper use of the words will make legal language solemn as well as sanctity, authority and rigor, such as whereby, wherefore, hereinafter, whereupon. Using these words can ensure one party or two parties of the contract, which will make the text accurate and increase the formality of legal documents. In order to express formality, we can use or, and, such as annul and set aside, entirely and completely remove, last will and testament, totally null and void, terms or conditions etc (Pontrandolfo, 2020). Meanwhile, in legal English, we can never use spoken English, such as we use "about, because of, call to meet, agreement, follow, in fact, temporary" instead of as regards, by virtue of, convene, ensue, in effect, interim.

\section{B. Accuracy}

Legal English has its own unique style, while the most important and most essential feature is accuracy. According to the principle of strict interpretation, in the application of law, written text is the sole basis for the judges to interpret legal documents. For the authority and seriousness of the legal text and the use of legal language under any circumstances, its expression should take accuracy as the basic guidelines and requirements (Giampieri, 2020). Therefore, legal language must be very accurate. For example, "no law or administrative or local rules and regulations shall contravene the constitution." The use of "shall" is absolutely accuracy.

\section{Ambiguity}

Ambiguity of legal language is that legal interpretation of certain provisions can not accurately refer to somebody or something, which is uncertainty. Generally it used to refer to legal facts that concerning the nature, scope, extent, number of cases or something that are unclear (J.C., 1965). Terms of degree such as: appropriate, take appropriate action, reasonable time. These words or phrases can not accurately express the meaning but they often play an important role in human mind.

\section{Professional}

Legal English is highly specialized and it is very difficult to master, it seems like a foreign language even to native English speakers, both the professional English and legal knowledge are required for the master of legal English. Speaking of the language itself, one of the reasons for it is difficult to master legal English is that legal English has a set of professional vocabularies that relatively complete, fully manifested legal profession. There are two types of these vocabularies.

One type is only used in specialized legal language and legal terms, but it is not used in other occasions (Sarcevic, 1997). For example, libel, appeal, due diligence, judicial system, tort, plaintiff, etc. It is very difficult to those readers who know little about law or know nothing about law.

The other is the general term with legal significance, we are familiar with these words, but when these common words are used in legal documents, they are no longer the ordinary meaning for the ordinary people to understand. There belong to legal category, their meaning are different from the original. Here are some commonly used words, in legal documents, only the legal professionals know them clearly. Such as action, avoid, counterpart, civil death, negligence, serve, party, plead, satisfaction.

\section{Difficulties of Translation of PROPER NOUNS IN LEGAl ENGLiSh}

Though proper nouns in legal English have their own characteristics, however, we need to obey translation rules when translating proper nouns in legal English. Generally speaking, names of people or places are transliterated, while 
names of institutions or books are semantic translated. Transliteration should follow the principle of conformity to the original and the principle of convention, the former is mainly applied to proper nouns of non-English origin. In pronunciation, these proper nouns' Chinese translation should be similar to the pronunciation of the original language as far as possible, but we can not translate them according to their pronunciation in English. While the latter concerning established wording or phrasing, no matter whether the translation is in accordance with the principle of conformity to the original, or there are other limitations, all we need to accept the translation instead of choosing another translation (Piszcz and Halina, 2020). Semantic translation requires accurate, we can not misunderstand the meaning of the original proper nouns. In a word, there are mainly three types of difficulties of translation of the proper noun in legal English.

First, how to translate the proper nouns to the adopt established popular translated names, those proper nouns are relatively easy to be pronounced and understood, transliteration according to the principle of conformity to the original and semantic translation based on meaning accurate are easy to be achieved, the difficulty mainly lies in how to translate to the adoption of established popular translated names. Some of this types of proper nouns are very familiar to us in legal English, they have been a name in Chinese for long, but for the translators who lack of legal knowledge will easily make mistakes according to pronunciation or literal meaning to translate, their translation will make the professional readers confused.

Next, to those proper nouns who comply with English pronunciation rule but without meaning in English, we also need to check whether these proper nouns have meaning or not in the original language. If any, transliteration or semantic translation should be decided in accordance with the situation. Basing on the English pronunciation and literal meaning directly to translate often leads to mistakes (Anna and Halina, 2020). To the kind of proper nouns originated from European language, what we mostly need to pay attention is whether there are general translated name in Chinese.

The last but not the least, names of books, institutions, official position also need to be determined their meaning in order to be semantic translated. What different from the second largest category is that this kind of proper nouns are more difficult to determine their pronunciation and meaning, translators even are unable to translate them in accordance with English pronunciation or literal meaning.

In a word, translation of the three main types of proper nouns in the stage of understanding and expressing have posed great challenges to translators, the translators indeed have also made many translation mistakes. For mistranslate of the translation of proper nouns, some scholars believes that, "there are few misunderstanding about it, however, there are many inappropriate expressions about it" (Pang, 2020, P.57), it seems that the view is incomplete correct in translation of the proper nouns in legal English.

\section{Methods of Translation of Proper Nouns in Legal English}

Speaking of the methods of translation, what just like is the commonly recognized "faithfulness, expressiveness and elegance" which was proposed by Yan Fu. It is the translation principles we should adhere to from macro perspective. We need to analyze it specifically when it comes to translation of the proper nouns in legal English. Firstly, we need to determine which to use between semantic translation and transliteration according to the word meaning in the sentence. To be specific, legal English proper nouns translators should insist on the following several methods.

\section{A. Transliteration}

Transliteration is both an important translation method of English nouns and basic translation method of names of people or places in proper nouns. The names of people or places and some new words in legal English can use transliteration to translate them. We are familiar with transliteration, for example, Hugo Grotius, Thomas Aquinas, John Austin etc, transliteration can be used to translate them.

1. Principle of Conformity to the Original

This is a generally accepted translation method, as a result of the mutual transmission of western culture and English's influence on various countries' language, many books are not translated into Chinese from the primitive works but re-translated the original version by taking English as the medium. For example, in German, German is "Deutsch", according to German pronunciation, it is "deyizhi"; Rome pronounces "Roma" in Italian, it should be translated into "luoma"; Japanese, Korean and other language translations cannot be in accordance with English pronunciation, we should take the Chinese character transliteration as the standard, such as: Okinawa, Osaka, Seoul, Pyongyang.

2. Adoption of the Established Popular Translated Names

Some of the translation has been standardized and universally spread and widely accepted by people, so there is no need for further change. For example, Russian "MockBa" should be translated into "Moscow" according to English pronunciation "Moscon"; in English, German "Muncher" should be translated into "Munica", while the Chinese translation is "Munich"; John, the Chinese translation is "Yuehan"; Johnson, the Chinese translation is "Yuehanxun". However, Johnathan actually should be translated into "Qiaonashuang", the latter is much closer to English pronunciation, but the former has already been socially recognized, so there is no longer any change for it.

\section{B. Omission}

Translators can choose omission while translating some of the proper nouns in legal English, that is copying the 
original text, but often need to add some qualitative terms before or after the original text, which makes the translation consistent with the expression of the target language, therefore the readers can understand the version fully at one glance (Jiang and Zhuang, 2019). For example, "the agreement is made and entered into by and between Party A and Party B", in the sentence, "made and entered into" and "by and between" are omitted.

\section{Annotation}

Proper nouns are the manifestation of culture, therefore translators may encounter difficulties resulting from cultural difference. At this time, translators may consider annotation if the above translation methods can not solve the problems.

Firstly, as the information receiver, in order to have a clearly understanding of the real meaning that the author want to convey, translators should fully understand the original culture that was loaded by proper nouns. Then, translators should add necessary background information by annotation so that the target version can convey adequate information to the readers, which can ensure the success of the inter-lingual communication (Giampieri, 2019). For example, "Yesterday, he read 'the Social Contract'," Suppose the readers do not know "the Social Contract", then the writer can annotate it to introduce more about the book, such as "the Social Contract, a book was written by French scholar Jean Jacques Rousseau".

\section{ReQuirements for Translators’ Translation of Proper NOUNS In LEGAL ENGLish}

How to deal with the challenge of understanding and expression? The author think that in order to translate the proper nouns in legal English better, firstly, we should be aware of the difficulties of translation of the proper nouns in legal English; secondly, we should try our best to accumulate extensive legal knowledge and improve our translation skills; thirdly, we should make full use of reference books and master the skills of Internet application; last but not the least, we should ask for advice from others (Dai, 2019).

\section{A. Awareness of the Difficulties}

In western countries, there is a tradition of neglecting the translation of proper nouns. Some scholars even think that "proper nouns never need to be translated, and there is no need to research on translation of proper nouns". Of course, it is relevant with the easiness of the translation and transliteration of the proper nouns in western language. Some scholars have made through discussion about it. In China, though few scholar have definitely indicate their contempt for translation of proper nouns, however, scholars who have focused on translation of proper nouns become less and less in recent years. It indicates that translation of proper nouns has not gained much attention by some of them (Hu, 2019). The few research on transliteration according to English pronunciation is the reason why some translators make mistakes. There are some proper nouns in common English dictionaries, however, some translators may make mistakes due to their despising, neglecting or not consulting dictionaries.

\section{B. Extensive Legal Knowledge and High Level of Translation Skills}

It has become the consensus in translation field that anyone who is engaged in professional translation must master basic professional knowledge. Naturally, anyone who is engaged in legal translation must be equipped with excellent legal knowledge. To the translation of the proper nouns in legal English, the main function of legal knowledge is to determine the general translated name of the well-known names of people, places, books, etc. Some of the proper nouns can not be found in dictionaries or other reference books. Although there are a variety of translations on the Internet, however, they may be unreliable. Under this condition, only by the translators' excellent legal knowledge could they finally determine the translation (Wallace and Nebot, 2019). What need to be pointed out is that legal knowledge is not limited to legal terms, but also includes politics, history, geography and other various kinds of knowledge which relates with law.

Of course, although the proper nouns' general translated name can be found in reference books or on the Internet, however, translators can determine the translated names if depending on their legal knowledge, it is undoubtedly that the time to consult dictionaries or surf the Internet can be omitted and the translators can raise their translation efficiency.

\section{Use of Reference Books}

Reference books of translators include linguistic dictionaries, professional knowledge dictionaries, translation exclusive dictionaries and other reference books. Translators should consult all the reference books as long as they have the conditions.

Firstly, the translators need to consult linguistic dictionaries frequently to see whether the linguistic dictionaries have collected the proper nouns encounter in translation. The translators can directly borrow the translated name if the proper nouns are collected by the dictionaries. It is noteworthy that, the translators can also check these nouns' respective language classification when translating those non-English proper nouns that directly borrowed or transferred in English.

Secondly, the translators also need to consult the professional dictionaries such as legal dictionaries to determine the translation of some legal terms (Ramos, 2019). It is necessary to consult translation exclusive dictionaries, especially 
the translation name dictionaries that are edited by Xinhua News Agency. We must consult them to ensure whether the translation is in line with national norms when translating name of places and people of the original version. Now there are also a lot of dictionaries which are edited by other organizations or individual, they also have the referential function to the legal translation, we should consult more reference books if we have the conditions.

\section{Skills of Network Applications}

Some scholars have dubbed the contemporary translators "the electronic person", the reason lies in computers and networks have been used widely in the entire process of translation. It is Natural legal translation with no exception. To the translation of the proper nouns in legal English, the role of network is mainly reflected in two aspects. On the one hand, it can help translators understand proper nouns in the original text. For example, to some legal terms, the translators can understand them by skimming the specific legal websites or searching related websites, consulting or reading discussions about them. To some proper nouns which we are uncertain about, we can also read the background information through the related websites to understand more about them and avoid mistranslating. On the other hand, it can determine the general translated name. Translators can determine the general translated name of many proper nouns through search engines. To those proper nouns that are not familiar by translators, but they are well-known by most people in legal field, we may determine its translated name completely through this method. Even those unknown proper nouns, sometimes there are test Chinese translation online for translators to choose from. The most commonly used search engine is Google. Input the English proper nouns and then set Chinese website search only, the high frequency presenting proper nouns are often the general translated name(Oțăt, 2018).It is certain that we can not totally accept all the contents on the Internet. The high frequency presenting proper nouns are not necessarily the general translated names, some are even the incorrect translated name, so translators should have certain judgment when seeking help to the network.

\section{E. Advice from Others}

Consulting the relevant experts becomes the only outlet when seek help to the reference books and the network still could not obtain the result, The consulting experts either are the teachers and friends in real life or anyone who comes from the virtual online world. The web forum is a good place for professional translators asking for advice to other people. It is also a sign for the translators to become "an electronic person". For some proper nouns transferred from other languages, the only way for translators to translate them is to consult other people (Flöter-Durr, 2018).

\section{CONCLUSION}

In fact, there is a method that can avoid the troubles in translation of proper nouns in legal English, which is omission. It means when using the original text, we do not need to translate the proper nouns into Chinese. Using this method, the translation will be full of different languages. It seems appropriate to some types of translation, such as computer science translation. It is not inappropriate for the translation full of different languages. Some people think that the translation full of different languages is a kind of fashion and they believe that it will become more and more commonly used. But the translation of the proper nouns in legal English has its own special solemnity. The readers in legal field are not as fashionable as the readers in computer science (Velykodska, 2018). As a result, the readers are not favor of the translation full of different languages in legal translation. Besides, translators will become lazy when adopting omission in translation of the proper nouns in legal English.

Translation of legal English can not neglect translation of proper nouns. And with the deepening of globalization, the sources of the proper nouns in legal English texts will become more complicated. The constituent of the proper nouns in legal English will become more diverse, which will give a bigger challenge to translators engaged in English-Chinese legal translation. The article has only made a shallow discussion in the hope of serving as a modest spur to induce someone to come forward with his valuable contributions and drawing more experts to pay more attention to translation of the proper nouns in legal English.

\section{REFERENCES}

[1] Anna Piszcz. Halina Sierocka. (2020). The Role of Culture in Legal Languages, Legal Interpretation and Legal Translation. International Journal for the Semiotics of Law-Revue internationale de Sémiotique juridique.33, 1-10.

[2] Congrong Pang. (2020). Three Principles of Convenience in Chinese Translation, Compilation and Reading of Legal Translation Works. Publication reference.6, 54-61.

[3] Catford, J.C. (1965). A Linguistic Theory of Translation. Oxford: Oxford University Press.

[4] Chris Wen-Chao Li. (2007). Foreign Names into Native Tongues. Target. International Journal of Translation Studies. 19, (1), 45-68.

[5] Diana Oțăt. (2018). Working with Bilingual Corpora to Enhance Legal Translation Competence. Romanian Journal of English. 1, (15), 134-144.

[6] Fernando Prieto Ramos. (2019). Cross-cutting methodological issues in legal translation. comparative Legilinguistics. 1, (37), 7-8.

[7] Gianluca Pontrandolfo. (2020). Testing Out Translation Universals in Legal Translation: Quantitative Insights From Patrizia Giampieri, The importance of Internet systematic search for legal translations. International Journal of Legal Discourse. 1, (5), 
83-102.

[8] Leping Zhang. (2008). The Characteristics and Application of Legal English, Hebei Legal Sience, (12),153-155.

[9] Ling Jiang. Yuyan Zhuang. (2019). Non-equivalence in Legal Translation. Theory and Practice in Language Studies (TPLS). 9, (12), 1630-1634.

[10] Minghui Li. (2009). On the Translation of Proper Nouns. Journal of the Party School of CPC Zhengzhou Municipal Committee. (5), 58-63.

[11] Miodrag M. Vukčević. (2020). Editorial preface and a short introduction to this issue's topic "Legal Translation and Interpreting in a Changing World: Technology - Outsourcing - Shifts". Babel. 66, (2), 163-171.

[12] Melissa Wallace. Esther Monzó Nebot. (2019). Legal translation and interpreting in public services: defining key issues, re-examining policies, and locating the public in public service interpreting and translation. Revista de Llengua i Dret-Journal of Language and Law. (71), 1-12.

[13] Margarete Flöter-Durr. (2018). Interpretation as a Pervading Problem in Legal Translation. Comparative Legilinguistics. 36, (1), 7-24.

[14] Newmark Peter. (2001). Approaches to Translation. Shanghai: Shanghai Foreign Language Education Press.

[15] Olena Velykodska. (2018). Legal Discourse: Text Analysis and Translation Strategies. Comparative Legilinguistics. 34, (1), 53-64.

[16] Piszcz Anna. Sierocka Halina. (2020). The Role of Culture in Legal Languages, Legal Interpretation and Legal Translation. International journal for the semiotics of law - Revue internationale de semiotique juridique. 33, (2), 3-10.

[17] Patrizia Giampieri. (2019). The BOLC for Legal Translations: A Trial Lesson. Comparative Legilinguistics. 39, (1), 21-46.

[18] Susan Sarcevic. (1997). New Approach to Legal Translation. The Hague, London, Boston: Kluwer Law International.

[19] Weifeng Hu. (2019). On Legal English Translation from the Perspective of Legal Linguistics. Review of Educational Theory. 2 , (3), 6-10.

[20] Xia Dai. (2019). Studies on the Legal Translation from the Perspective of Legal Pluralism. Theory and Practice in Language Studies (TPLS).9, (8), 973-977.

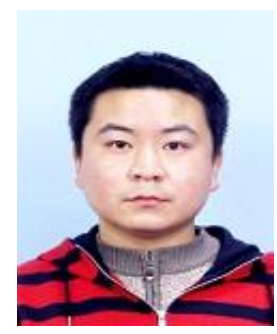

Gang Tang was born in Shaoyang City, Hunan Province, P.R. China in 1987. He received his Bachelor degree in Foreign Language and Literature, LL.B. from Wuhan University of Science and Technology, P.R. China in 2011. He received his Master degree in International Laws from Kunming University of Science and Technology, P.R. China in 2015.

$\mathrm{He}$ is currently a Ph.D. Candidate in Wuhan University Institute of International Law, Wuhan, P.R. China. His research interests include international law and legal English. 